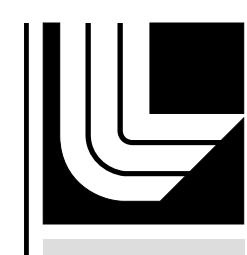

LAWRENCE LIVERMORE N A T IO N A L LABORATORY

\title{
Wavefront control for the Gemini Planet Imager
}

\section{UCRL-CONF-220694}

Lisa A. Poyneer, Jean-Pierre Veran, Daren Dillon, Scott Severson, Bruce Macintosh

April 19, 2006

Advances in Adaptive Optics II

Orlando, FL, United States

May 24, 2006 through May 31, 2006 
This document was prepared as an account of work sponsored by an agency of the United States Government. Neither the United States Government nor the University of California nor any of their employees, makes any warranty, express or implied, or assumes any legal liability or responsibility for the accuracy, completeness, or usefulness of any information, apparatus, product, or process disclosed, or represents that its use would not infringe privately owned rights. Reference herein to any specific commercial product, process, or service by trade name, trademark, manufacturer, or otherwise, does not necessarily constitute or imply its endorsement, recommendation, or favoring by the United States Government or the University of California. The views and opinions of authors expressed herein do not necessarily state or reflect those of the United States Government or the University of California, and shall not be used for advertising or product endorsement purposes. 


\title{
Wavefront control for the Gemini Planet Imager
}

\author{
Lisa A. Poyneer ${ }^{a}$, Jean-Pierre Véran ${ }^{b}$, Daren Dillon $^{c}$, Scott Severson ${ }^{c}$, and Bruce A. \\ Macintosh $^{a}$ \\ ${ }^{a}$ Lawrence Livermore National Lab, 7000 East Ave, Livermore, CA, 94550 \\ ${ }^{b}$ Herzberg Institute of Astrophysics, 5071 West Saanich Road, Victoria, British Columbia, \\ Canada V9E2E7 \\ ${ }^{c}$ UCO Lick Observatory, Laboratory for Adaptive Optics, University of California, Santa Cruz, \\ 1156 High Street, Santa Cruz, California, 95064
}

\begin{abstract}
The wavefront control strategy for the proposed Gemini Planet Imager, an extreme adaptive optics coronagraph for planet detection, is presented. Two key parts of this strategy are experimentally verified in a testbed at the Laboratory for Adaptive Optics, which features a $32 \times 32$ MEMS device. Detailed analytic models and algorithms for Shack-Hartmann wavefront sensor alignment and calibration are presented. It is demonstrated that with these procedures, the spatially filtered WFS and the Fourier Transform reconstructor can be used to flatten to the MEMS to $1 \mathrm{~nm}$ RMS in the controllable band. Performance is further improved using the technique of modifying the reference slopes using a measurement of the static wavefront error in the science leg.
\end{abstract}

Keywords: wavefront control, fourier reconstruction, adaptive optics, high-contrast imaging

\section{INTRODUCTION}

The Gemini Planet Imager (GPI) ${ }^{1}$ is a proposed Extreme Adaptive Optics Coronagraph which will enable direct imaging of extrasolar planets from the ground-based 8-meter Gemini telescope. The science-driven requirements for Adaptive Optics (AO) wavefront control are challenging and go beyond what has been accomplished by present AO systems. To met these requirements, several new technologies and algorithms are being developed and tested for GPI. These new items include the spatially filtered wavefront sensor (SFWFS) ${ }^{2}$ (which prevents aliasing and enables much-improved phase compensated in the controllable spatial frequency band of the $\mathrm{AO}$ system ${ }^{3}$ ), the computationally efficient wavefront reconstruction method Fourier Transform Reconstruction (FTR $)^{4}$ and its optimal, modal version Optimal Fourier Control (OFC), ${ }^{5}$ the use of microelectromechanical systems (MEMS) mirrors for high-order wavefront compensation, and the use of a high-precision interferometric calibration system in the science leg of the instrument to provide real-time information about static residual errors. ${ }^{6}$ This paper first details the complete wavefront control scheme that is being developed for GPI. Then it focusses in on two important aspects of this scheme which are being experimentally validated at the Laboratory for Adaptive Optics (LAO) extreme AO testbed: the ability to control a high-order MEMS mirror to $1 \mathrm{~nm}$ RMS error in band with the SFWFS and FTR, and the ability to manipulate and improve the wavefront control performance at the nm-level using calibration input from the science leg.

\section{GPI SYSTEM DESIGN}

\subsection{System block diagram}

Fig. 1 shows a slightly simplified block diagram of the GPI wavefront control system. Most of the multiple correction elements have been omitted for clarity (more on these below). The diagram can be divided into three separate parts: obtaining slopes, reconstruction of slopes to desired phase correction, and determining how to place that phase on the DMs.

Send correspondence to Lisa Poyneer: poyneer1@llnl.gov, 19254233360 


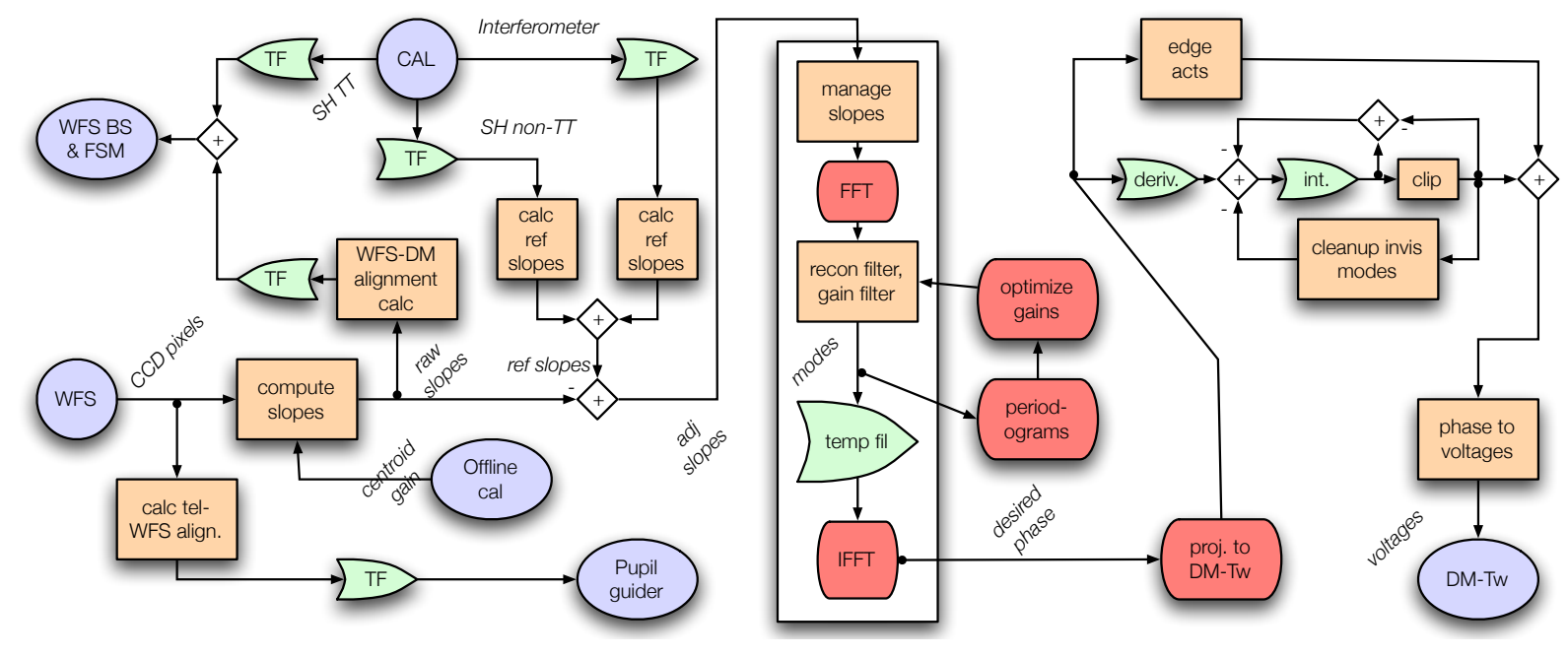

Figure 1. Wavefront control block diagram. Only one DM (the tweeter) is shown for clarity. Supervisory processes using information from the WFS and from the calibration system at the science camera adjust the performance by moving optics and modifying reference slopes. Phase estimation is done with Optimal Fourier Control. Control of the DMs includes invisible mode cleanup, control of edge actuators and voltage conversion.

The process of obtaining the slopes involves both the measurement of the atmosphere with the WFS and the modification of those slopes based on calibration information. GPI uses a spatially filtered Shack-Hartmann wavefront sensor. This WFS will be sized to match the WFS lenslet array and will have 44 subapertures across the diameter $D=8 \mathrm{~m}$ pupil, giving a subaperture size and actuator spacing of $d=18.2 \mathrm{~cm}$. A square field stop of angular size $\lambda / d$ in the focal plane before the lenslet array filters out spatial frequency components beyond the sensor's cutoff frequency and limits aliasing. The Shack-Hartmann lenslets form an array of spots on the WFS CCD. These spots will have angular size 1.8 arcsec and will be $2 \times 2$ pixels each on the CCD. The slopes will be computed from the CCD image; the baseline algorithm is the standard quadcell centroid algorithm, though more sophisticated algorithms have been proposed. ${ }^{7}$ The WFS will be initially aligned using slope information; this is a major research topic at the LAO and is detailed in Section 5.

The slopes are processed before being used in phase reconstruction. The first processing step is to adjust (if necessary) for the spot size. The quadcell is susceptible to gain changes due to spot size changes induced by the atmosphere. ${ }^{8}$ However, use of the spatial filter should prevent the spot size changes ${ }^{9}$ that result in varying centroid gain. An offline system is planned to evaluate any gain changes that do occur. The most significant modification of the raw slopes, however, is with references.

A set of reference slopes is subtracted at each time step from the raw slopes. These reference slopes are used to compensate for any static or quasi-static wavefront error seen by the science camera. In GPI the reference slopes will be set prior to operation, but will be monitored and modified during actual observation. A dedicated calibration system unit is included in GPI after the coronograph. It will provide the wavefront control system with an estimate of the static phase error seen by the science camera at the science wavelength (which is different than the WFS wavelength). This information, which will be provided at an effective rate of $1 \mathrm{~Hz}$, will be converted into reference slopes or optics motions. The beamsplitter and steering mirror can correct for both pupil motion and spot translation. It is anticipated that the raw slope signals will be used to adjust these optics if necessary, though more work remains to be done on exact methods. Proper use of information from the calibration system to improve wavefront quality is an essential portion of the GPI strategy. Section 7 discusses our progress in validating the reference-slope manipulation strategy for changing the phase error seen by the science camera.

Once the slopes are processed, they are sent to the reconstruction unit, which uses the OFC algorithm. At each time step, the phase is estimated from the slopes using FTR. This method is computationally efficient and uses the Fourier modes as its natural basis set. The optimization of OFC uses temporal estimates of signal 


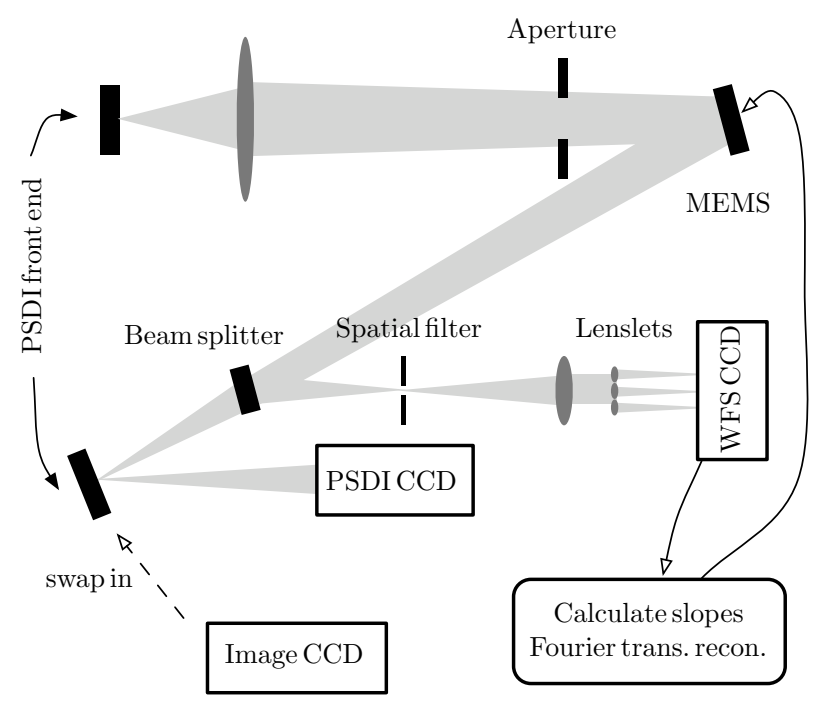

Figure 2. PSDI-WFS testbed to control $32 \times 32$ MEMS device.

and noise strength to determine the optimal temporal control to minimize the residual error power. Since the reconstruction is done in the Fourier basis set, specific locations in the PSF of the system are optimized, exploiting the PSD-PSF relationship. ${ }^{10,11}$ The temporal filter (i.e. the integrator-gain control law used in the closed-loop operation) is done in the frequency domain. This process will give the estimate of the phase which needs to be compensated by the correction elements. In this way our strategy is similar to other estimation-fitting approaches, such as that for minimum-variance wavefront reconstruction. ${ }^{12}$

GPI will need to use up to five mirrors for phase compensation. As specified by Gemini, the telescope primary M1 and secondary M2 will receive modal information slowly. The real-time control system will use a high-order MEMS device (the Tweeter), a low-order but high stroke DM (the Woofer) and potentially a separate Tip-Tilt mirror. Given the desired phase compensation output from OFC, the control system will split the phase up amongst the mirrors. We are currently researching how best to divide the commands (e.g. in spatial frequency space or actuator space) and whether to divide them based on temporal frequencies as well. This work will follow on research done for tip-tilt mirror control. ${ }^{13}$ The tip-tilt mirror will correct those two modes. To first order, the Woofer will take the high-amplitude, low-spatial frequency (and perhaps low-temporal frequency) modes and the Tweeter will compensate everything else.

For each mirror the temporal control involves several steps. Fig. 1 shows the Tweeter process as an example. For the Tweeter, the area around the active pupil needs to be controlled in a sensible fashion, via slaving or some other method, as represented by the 'edge acts' box in the diagram. Invisible mode cleanup is done in residual update space as opposed to integrated open-loop phase space. The cleanup is done based on results of actuator clipping, which is due to the maximum stroke possible on the MEMS. ${ }^{14}$ After the edge control clipping and cleanup, the final phase for the Tweeter is converted into the necessary voltages to move individual actuators.

\section{EXPERIMENTAL TESTBED}

Our high-contrast imaging testbed has been described in detail elsewhere. ${ }^{15}$ A schematic of the setup is shown in Fig. 2. The system consists of a phase-shifting diffraction interferometer (PSDI) which has sub-nanometer measurement accuracy. ${ }^{16}$ The MEMS device is a $32 \times 32$ Boston Micromachines continuous face-sheet deformable mirror which has an actuator spacing on the device of $d=340$ microns. The MEMS is driven with custom 13-bit electronics and has a maximum stroke of approximately 1-micron deflection. A beam splitter allows simultaneous 


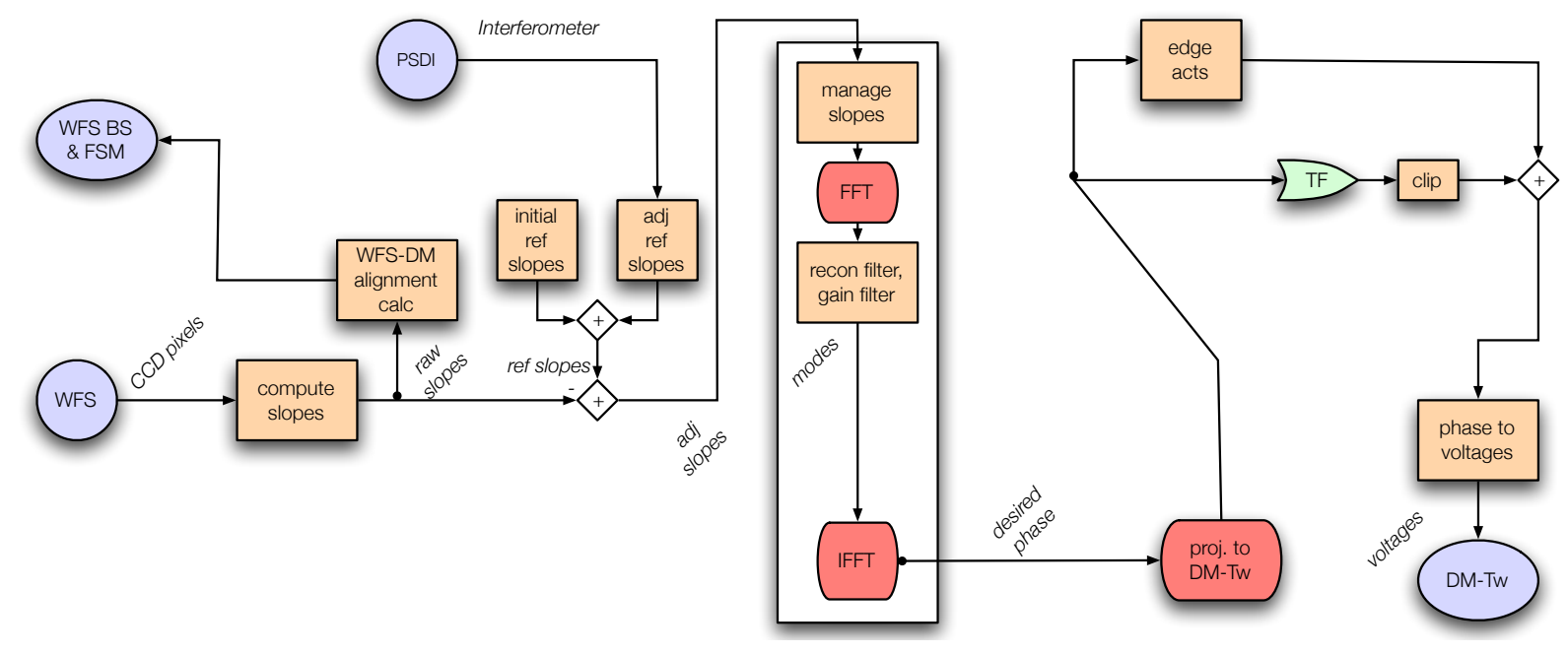

Figure 3. Simplified Wavefront control block diagram showing relevant parts that are implemented in the LAO testbed. Alignment and calibration are done off-line between closed-loop runs.

measurement by the PSDI and the WFS. In PSDI mode, the beam is interfered at focus with a reference wavefront, then imaged by the PSDI CCD and numerically back propagated to the MEMS plane.

As mentioned above, the WFS is spatially-filtered and has a square field stop. The new Vitrum lenslet array produces spots on the WFS CCD with $16 \times 16$ pixel subimages and a pixel size of 0.65 of a Nyquist pixel. The slopes are calculated from the spots using a correlation algorithm. ${ }^{17}$ When a physical aperture is used (as shown in the figure), the WFS calibration code determines which subapertures have adequate illumination. When a software aperture is used, the valid subapertures are determined by a mapping from the software aperture onto the lenslet array. Edge subapertures are considered valid if the center of the subaperture is inside the edge of the software aperture; otherwise the subapertures are not used. FTR automatically adjusts to the present aperture configuration. After dereferencing, the slopes are reconstructed with FTR. After temporal filtering with the control law, the desired actuator phase displacements are used to slave uncontrolled actuators then converted to volts via pre-determined calibration.

As currently configured the testbed incorporates part of the full wavefront control system model. The subset is shown in Fig. 3. In the testbed the WFS and PSDI operate at the same wavelength. The PSDI has the role of the calibration system in the science leg. Initial alignment of the WFS optics is done by hand, and is discussed in detail in Section 5. Because the phase aberration is static (at present simply the non-flat shape of the MEMS device) the reconstruction stage does not use any temporal optimization. The MEMS control has all of the features planned for GPI except modal cleanup, which are not yet necessary.

\section{PROPAGATION OF SYSTEM ERRORS}

Static and quasi static errors in the science camera need to be minimized in GPI. Because these slowly varying errors do not average out (like errors due to WFS noise) they inhibit planet detection. These errors can be mitigated to some extent by imaging and data analysis techniques. These techniques can use multiple simultaneous images at different wavelengths, use images with different telescope field rotations or use reference stars. Marois et $a l^{18}$ has tested the these approaches at CFHT. Science camera errors can also be reduced through manipulation of the AO control system, specifically through the reference slopes. Use of reference slopes allows the $\mathrm{AO}$ system to be driven to create specific phase aberrations in a way independent of the reconstruction algorithm.

An excellent discussion of how errors in the science leg and WFS leg affect each other in given in Section 3C of Véran and Herriot $;^{8}$ we follow their notation here in applying that analysis to our system. The AO system is 
divided into three portions. The common path is the portion that both the WFS and the science camera (which in our case is the PSDI) see. The phase aberration in the common path is given by $\mathbf{m}_{\text {com }}$ and in Fig. 2 it consists of phase aberrations from the fiber launch, the MEMS and up to the surface of the beam splitter.

The additional phase aberration seen by the PSDI but not by the WFS is $\mathbf{m}_{\mathrm{im}}$; in this case it is the phase error induced by transmission through the beam splitter. The phase aberration seen only by the WFS is $\mathbf{m}_{\mathrm{wfs}}$. This includes two portions. The physical phase error component is due to actual optical aberrations, such as the phase error of reflection on the beam splitter and flaws in the WFS optics. The virtual phase component is that which arises from misalignments of the measurement apparatus which propagate through the system, inducing phase errors. These are in particular due to the alignment of the CCD, which is discussed in detail in Section 5.2.

If the WFS closed-loop system is run with out reference slopes, the phase aberration seen by the PSDI is driven to $\mathbf{m}_{\mathrm{im}}-\mathbf{m}_{\mathrm{wfs}}$. The common phase $\mathbf{m}_{\mathrm{com}}$ has been corrected, but a static error term is present in the science leg. This can be removed through proper referencing. Ideally, the phase $\mathbf{m}_{\mathrm{wfs}}-\mathbf{m}_{\mathrm{im}}$ is subtracted from the slopes as references. This will cause the WFS closed-loop system to drive the MEMS such that the science camera sees no phase aberration. In reality, this reference offset must be measured in some manner in the actual system. This is typically done with a probe source and image sharpening. ${ }^{19}$

In our system this is done by flattening the MEMS with the PSDI as a direct-phase sensor. The MEMS is driven in closed-loop to a shape $-\mathbf{m}_{\mathrm{com}}-\mathbf{m}_{\mathrm{im}}+\mathbf{e}_{\mathrm{psdi}}$. The PSDI does not see zero phase; is sees a residual error $\mathbf{e}_{\mathrm{psdi}}$ of typically $0.6 \mathrm{~nm}$ RMS in band. ${ }^{20}$ The MEMS is held with this shape and the WFS takes a slope measurement. In practice, our reference slopes still have some level of error on them, due to transients such as WFS noise and air currents on the bench. This process results in a reference phase (in slope space) of $\mathbf{m}_{\mathrm{wfs}}-\mathbf{m}_{\mathrm{im}}+\mathbf{e}_{\mathrm{psdi}}+\mathbf{e}_{\mathrm{ref}}$.

When the WFS system is run with these references, it converges to a residual phase error as seen by the PSDI of $\mathbf{e}_{\text {psdi }}+\mathbf{e}_{\text {ref }}$. In order to reach $1 \mathrm{~nm}$ RMS in-band in our present configuration, $\mathbf{e}_{\text {ref }}$ must be less than $0.8 \mathrm{~nm}$ RMS. In the following sections we describe in detail the process of aligning the WFS sufficiently such that this level of performance can be achieved.

\section{ALIGNMENT OF THE WFS}

Alignment of the WFS is particularly important in our design because we use FTR. FTR assumes that the WFS slope measurements are on a square grid, with the MEMS actuators exactly co-incident with the junctions of the WFS lenslets. In practice, this will not be exactly the case; some misalignments, however small will exist. In a matrix-based AO system, the system control matrix can be measured directly through a process of activation of actuators and WFS measurements. The resulting control matrix will contain information about the system alignment and should operate well. In fact, AO systems can be run in a non-matching geometry such as with over-sampled Shack-Hartmann sensor and a pseudo-hexagonal actuator geometry DM. ${ }^{21}$

To ensure the best possible alignment we rely on analytic models. These models allow us to quantify the amount of misalignment in the system and asses its impact on performance. These methods are incorporated into alignment procedures for the optics themselves to provide as good alignment as possible.

\subsection{WFS optics aberrations}

There do exist aberrations in the WFS leg that cannot be removed through alignment. For example, phase aberrations in the relay optics or individual flaws in the the lenslets. As of now, these errors can only be compensated for by measuring them accurately and using their values in the reference slopes. In the testbed these errors are $13 \mathrm{~nm}$ RMS in-band.

\subsection{Alignment of WFS lenslets to the WFS CCD}

The WFS lenslets produce an array of spots on the WFS CCD. If these spots are not precisely aligned to the WFS CCD pixels, the slopes which are calculated will be offset by a bias. This bias will lead to incorrect phase reconstructions unless it is either aligned or calibrated out.

The errors induced by lenslet-CCD misalignment are easiest to measure and deal with when the MEMS is shaped to compensate for any $\mathbf{m}_{\text {com }}$, producing a flat wavefront into the WFS leg. In order to determine the 
impact of alignment errors, we need to have specifications for the WFS leg. In particular, the WFS pixel size is very important, because it sets the gain from spot motion to phase slope. A Nyquist-sampled spot has a pixel size $\lambda /(2 d)$, where $\lambda$ is the wavelength of light in the WFS and $d$ is the subaperture diameter. The pixel size is expressed as a ratio to this Nyquist size, as $s$ is the actual angular pixel size divided by $\lambda /(2 d)$. From this the conversion from pixels of spot motion to phase slope is easily determined by Fourier Optics. Given a spot shift in pixels of $x$, the phase slope in $\mathrm{nm}$ across a subaperture is

$$
\Delta_{\phi}=x \frac{s \lambda}{2} .
$$

Also important is the pitch of the spot array on the CCD, which is the number of pixels between the centers of adjacent spots, assuming no aberrations are present. This pitch is given by the variable $P$. Ideally this pitch $P$ is a whole number of pixels. We will assume that given the pitch $P$, each spot has a $P \times P$ pixel region which is used in slope computation.

The misalignment of the WFS lenslet spot array to the CCD is modeled as a function of five parameters. The CCD can be translated in-plane along the $\mathrm{x}$ - and y-axes, producing translations $T_{x}$ and $T_{y}$ in units of pixels. The $\mathrm{CCD}$ can be rotated in plane by an angle, $\theta$. Finally, the spot array can be magnified along either the $\mathrm{x}-$ and y-axes. This physically translates to the CCD being off of perpendicular to the optical axis. Given this, magnification ratios $M_{x}$ and $M_{y}$ are always less than 1 . We will assume the the CCD is at the correct distance from the lenslet array such that spots are all in focus.

Translation of the CCD relative to the spot array is the easiest error to analyze and fix. The translation leads to constant slopes for that axis. This translates to tip and tilt in the science leg. If we tolerance the system to allow only a certain level of RMS error in the science leg, we can determine analytically how much uncompensated translation is allowed. Setting a limit of $\sigma_{\text {trans }}$ for the RMS error due to translation along one axis, the maximum shift is

$$
T_{x}=\frac{8 \sigma_{\text {trans }}}{s \lambda \hat{N}}
$$

pixels. Setting a limit of $10 \mathrm{~nm}$ RMS, the maximum translation for the LAO testbed is $T_{x}=8.6$ millipixels. For the GPI setup the translation is $T_{x}=0.57$ millipixels. As long as the overall spot motion is small, the translation in either axis will only introduce a bias and not change the gain of the WFS. As such, this error can be completely removed using reference slopes which correctly measure $T_{x}$ and $T_{y}$. So this tolerance sets the error of the reference slopes off the true translation.

Magnification of the spots onto the pixels of the CCD results in a contraction or expansion of the spot array, which is a linear slope term. Magnification along the $\mathrm{x}$-axis only effects the $\mathrm{x}$-slopes and likewise magnification along the $y$-axis changes only the y-slopes. This linear slope signal results in a second-order phase term being seen by the science camera of the form

$$
\phi_{\mathrm{sci}}(x, y)=-\frac{s \lambda}{2}\left[P\left(M_{x}-1\right) \frac{x^{2}}{2}+P\left(M_{y}-1\right) \frac{y^{2}}{2}\right] .
$$

Magnification along only one axis reconstructs to a parabolic cylinder. Equal magnifications reconstruct to focus; opposite amount scalings reconstruct to astigmatism. As above, tolerances can be set. If only one axis has magnification (e.g. the x-axis) then the allowable magnification range for a given RMS phase error $\sigma_{\text {mag }}$ in the science leg is

$$
\left|M_{x}-1\right|<\frac{64 \sigma_{\mathrm{mag}}}{s P \lambda \hat{N}^{2}}
$$

Setting a limit of $10 \mathrm{~nm}$ RMS, for the LAO testbed this range is $1.6 \times 10^{-4}$, or $0.99984<M_{x}<1.00016$. For GPI this range is even tighter: $5.16 \times 10^{-5}$. This major impact (the quadratic phase) of magnification can be removed with accurate referencing. There is a secondary effect of the spot motions being scaled by the magnification. This results in a change in the WFS gain. For the scales of magnification involved in our setups, the gain change is negligible. 
The final misalignment is rotation of the CCD relative to the spot array. This projects the true slopes into a basis set which is slightly rotated, leading to additional error, especially around the edges of the pupil. The rotation is exacerbated when the field of view $s P$ of each subimage and the number of subapertures $N$ become large. Unlike the previous two cases, there is not a simple relationship between the offset slopes caused by the misalignment and a phase aberration. These offsets do not fully reconstruct to a Zernike mode. Instead, a portion of the slope signal is in the null space of the reconstructor and does not reconstruct to a phase. What is reconstructed goes into Zerinke mode 15. This error cannot be completely eliminated by the use of references. This is because the rotation actually projects the spot motion into a different basis set, which is rotated from the true pixel $\mathrm{x}$ - and y-axes. As a consequence, this rotation error should be as close to zero as possible.

All five of these parameters are estimated from raw WFS slopes by measuring the slopes with a flat shape on the MEMS and then evaluating the constant, linear and rotational portions of the slopes.

\subsection{Impact of misalignment of lenslets to CCD}

As shown above, the conversion factors from reference slopes to phase errors for both the LAO testbed and GPI mean small errors can produce significant phase. To verify the factors in the LAO, and to check to what level we could actually fix these errors, we systematically added focus to the system.

First we took references from a PSDI closed-loop run and then flattened the MEMS with the WFS. This provides a baseline set of references and a baseline error as seen by the PSDI. Then linear slopes for both $\mathrm{x}$ and $\mathrm{y}$ were added to the references, and the closed loop was run to convergence. A PSDI measurement was taken of the residual error. Subtracting out the baseline error signal should produce a pure focus, plus a low level of noise. We began this test by adding in slopes that had a change in value of 5 millipixels from one subaperture to the next. Using the system parameters, this converts to a $24 \mathrm{~nm}$ RMS phase error (all freqs) in the science leg. This was confirmed by the first measurement. (In fact, this protocol was used to verify the pixel size of the WFS, which is used in the slope calculation to convert from pixels of spot motion to $\mathrm{nm}$ of phase slope.)

This process was repeated and at each iteration the amount of focus was halved. This method produced clean focus signals as seen by the PSDI down to a slope difference of 0.625 millipixels, which is $3 \mathrm{~nm}$ RMS focus. For 0.3125 millipixels, which is $1.5 \mathrm{~nm}$ RMS of focus, other errors exist in the residual phase of similar size, making the addition of focus not exact. From this test follow two conclusions. First, we can manipulate the phase as seen in the science leg systematically by modifying the slopes, even down to levels of a few nm RMS error. Second, the very small differences in reference slope values (on the order of a millipixel) emphasizes the need to have very accurate references.

\subsection{Alignment MEMS to the WFS lenslets}

Whereas most of the CCD misalignments simply added a phase term which can be removed by proper referencing, misalignments between the actuators and the lenslets mean that the WFS measurements are not where FTR assumes them to be.

To start the analysis we begin with five parameters which describe the way the actuator and lenslet grids are aligned to each other. The grids can be rotated relative to each other by angle $\theta$, the spacing of the actuators can be magnified or demagnified along either axis relative to the lenslets by ratios $m_{x}$ and $m_{y}$, and the actuator grid can be translated in either $\mathrm{x}$ or $\mathrm{y}$ from the lenslets by translations $x_{0}$ and $y_{0}$, where the distance between lenslets is 1 . These misalignments represent physical displacements of the actual optics themselves, and as such can be adjusted.

An approach to aligning the actuators to lenslets is to place waffle on the MEMS and then adjust the WFS until the observed slopes are as close to uniform zero as possible. ${ }^{22}$ We desire, however, a more quantitative method based on an analytic treatment of the situation. We have developed an algorithm that estimates the magnifications and translations using WFS measurements of two different signals on the MEMS. At this time rotation is addressed separately. Given a spatial frequency pair $k, l$, we place on the MEMS a phase cosine. This will (as long as neither $k$ nor $l$ equal 0 or $N / 2$ ), produce the following surface displacement on the MEMS mirror as seen by the spatially filtered WFS,

$$
\phi(x, y)=A \cos \left(\frac{2 \pi}{N}[k x+l y]\right)
$$


where $A$ is a scaling constant and we have assumed that the actuator spacing $d$ is 1 . We can incorporate translation and magnification along the axes as

$$
\phi(x, y)=A \cos \left(\frac{2 \pi k\left[x-x_{0}\right]}{N m_{x}}+\frac{2 \pi l\left[y-y_{0}\right]}{N m_{y}}\right),
$$

where $x_{0}$ and $y_{0}$ are the translations in fractions of a subaperture and $m_{x}$ and $m_{y}$ are magnifications. We further assume that the magnification is defined as coming from the center of the actuator array, giving our variables a shift of $N / 2$. Using the ideal response of the Shack-Hartmann WFS, the WFS slope signals that are observed are

$$
\begin{aligned}
& x[m, n]=\frac{A N m_{y}}{2 \pi l}[ \sin \left(\frac{2 \pi k\left[m-N / 2-x_{0}\right]}{N m_{x}}+\frac{2 \pi l\left[n-N / 2-y_{0}\right]}{N m_{y}}\right) \times \\
&\left\{\left[\cos \left(\frac{2 \pi k}{N m_{x}}\right)-1\right]\left[\cos \left(\frac{2 \pi l}{N m_{y}}\right)-1\right]-\sin \left(\frac{2 \pi k}{N m_{x}}\right) \sin \left(\frac{2 \pi l}{N m_{y}}\right)\right\}+ \\
& \cos \left(\frac{2 \pi k\left[m-N / 2-x_{0}\right]}{N m_{x}}+\frac{2 \pi l\left[n-N / 2-y_{0}\right]}{N m_{y}}\right) \times \\
&\left.\left\{\sin \left(\frac{2 \pi k}{N m_{x}}\right)\left[\cos \left(\frac{2 \pi l}{N m_{y}}\right)-1\right]+\sin \left(\frac{2 \pi l}{N m_{y}}\right)\left[\cos \left(\frac{2 \pi k}{N m_{x}}\right)-1\right]\right\}\right],
\end{aligned}
$$

and

$$
y[m, n]=\frac{m_{x}}{k} \frac{l}{m_{y}} x[m, n] .
$$

We pick a specific mid-range spatial frequency and apply cosines for that pair $k, l$ and the switch $l, k$. This ensures that there is no differential scaling due to spatial frequency location, since the WF and MEMS frequency responses should be symmetric. Given these measurement, $x_{1}[k, l], y_{1}[k, l]$ and $x_{2}[k, l], y_{2}[k, l]$, we then estimate the parameters using the conjugate gradient technique, minimizing the error function

$$
\begin{aligned}
J=\sum_{m, n \in S}\left(x_{1}[m, n]-\hat{x}_{1}\left[m, n, \hat{x}_{0}, \hat{y}_{0}, \hat{m}_{x}, \hat{m}_{y}\right]\right)^{2}+\left(y_{1}[m, n]-\hat{y}_{1}\left[m, n, \hat{x}_{0}, \hat{y}_{0}, \hat{m}_{x}, \hat{m}_{y}\right]\right)^{2}+ \\
\left(x_{2}[m, n]-\hat{x}_{2}\left[m, n, \hat{x}_{0}, \hat{y}_{0}, \hat{m}_{x}, \hat{m}_{y}\right]\right)^{2}+\left(y_{2}[m, n]-\hat{y}_{2}\left[m, n, \hat{x}_{0}, \hat{y}_{0}, \hat{m}_{x}, \hat{m}_{y}\right]\right)^{2} .
\end{aligned}
$$

Our estimated parameters are denoted with hats $\left(\hat{x}_{0}\right)$ and our estimates of the slopes (based on the above formulas) are given by hats as well $(\hat{x}[m, n])$. This technique is quite accurate in our simulation codes. In practice on the testbed the estimates do suffer from noise, especially for $x_{0}$ and $y_{0}$. This can be ameliorated, however, with more temporal averaging.

\subsection{Impact of misalignment of actuators to lenslets}

If the lenslets are misaligned to the actuators, the WFS will measure the phase at slightly the wrong location. How much error is present is entirely dependent on the signal content of the phase aberration $\mathbf{m}_{\text {com }}$ which the system is trying to correct. As such, no easy formulas such as those in Section 5.2 can be provided here.

We have been able to develop a reasonably useful model for the translation errors $x_{0}$ and $y_{0}$. Simple translations are multiplies by complex exponentials in the Fourier domain. This means that the gain of the AO system for a specific spatial frequency is modified through multiplication by a complex number. This will move the location of the closed-loop pole, changing the shape of the frequency response. An uncorrected translation misalignment will reduce temporal bandwidth in the dynamic aberration case, but in the static aberration case it will have little impact until a certain point, when performance will start to break down. The testbed optical setup allows an easy adjustment of the y-position of the MEMS device.

To evaluate the importance of proper alignment, the testbed was systematically misaligned in translations along y-axis. Before this began, the MEMS was flattened with the PSDI and reference slopes were taken. Then for several runs the MEMS was moved and the alignment algorithm described above was used to estimate 


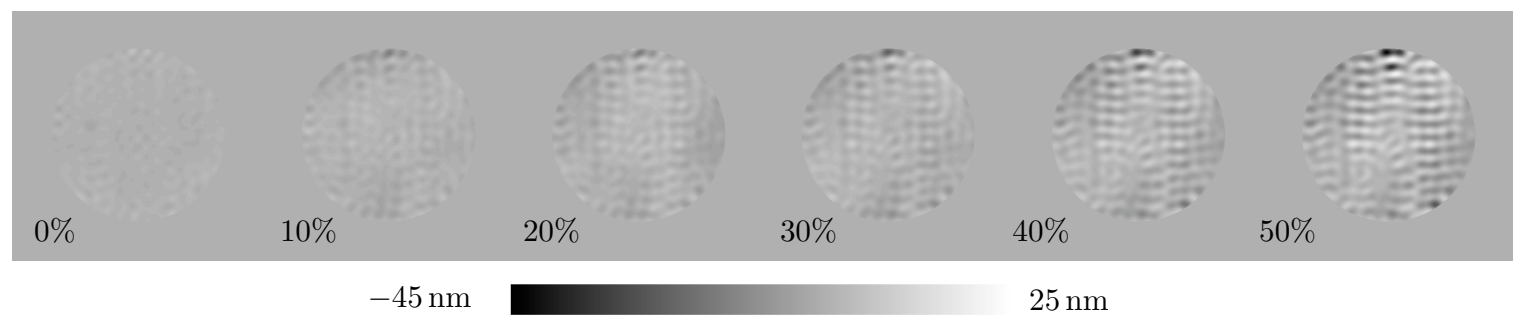

Figure 4. Low-pass filtered extra phase errors from WFS-FTR operation when the actuators are misaligned from the lenslets. Percentage of a subaperture misaligned in y given in figure. The system remains stable, but converges to the wrong shape, with substantial phase power along the $y$-frequency axis.

the misalignment. For each misalignment, the WFS was run in closed loop to convergence without changing references. and PSDI measurements were taken. The level of additional error was obtained by subtracting off the PSDI-flattened residual. These additional errors are shown in Fig. 4. As the system becomes more mis-translated in $\mathrm{y}$, strong error terms which are of high spatial frequency in $\mathrm{y}$ but very low in $\mathrm{x}$ are introduced. For each additional $10 \%$ of a subaperture that the system is misaligned by along y, an extra 1.8 to $2.0 \mathrm{~nm}$ RMS in-band is added to the residual error. This is just for flattening the MEMS. More tests on this and other misalignments will be conducted with phase plates in the system to provide a substantial $\mathbf{m}_{\text {com }}$ to correct. We have not been able to easily test the other misalignment parameters easily, due to the optical design and mounts.

\section{PERFORMANCE RESULTS WITH REFERENCE SLOPES}

A major goal for our validation of new GPI techniques is to demonstrate that the MEMS mirror can be controlled to have a surface with $1 \mathrm{~nm}$ RMS error in band. Extensive work has been done by our colleagues in detailing the challenges of the working with our MEMS device ${ }^{23}$ and in fundamental system limitations when using the PSDI as a direct-phase sensor to flatten the MEMS. ${ }^{20}$ Readers seeking detailed discussions on matters such as the voltage calibration of the MEMS or the various ways in which an actuator misbehaves should consult those papers. We will focus here on the WFS leg and FTR.

Our operation protocol begins with a precise alignment of the WFS leg using the tools described above. These alignment tools reported a misalignment of the spots to the CCD of: translations $T_{x}=-0.06$ pixels, $T_{y}=-0.05$ pixels, magnifications of $M_{x}=1.00045$ and $M_{y}=1.00035$ and a rotation of $\theta=-2$ millidegrees $(7.2 \operatorname{arcsec})$. This CCD misalignment, if uncompensated for by the references, is $27 \mathrm{~nm}$ RMS in-band, excluding tip and tilt. The actuators-lenslet alignment tool reported translations of $x_{0}=-0.90 \%$ of a subaperture and $y_{0}=-1.25 \%$ of a subaperture. The magnifications are $m_{x}=1.0038$ and $m_{y}=1.002$. The PSDI was used to flatten the MEMS with a 9.2-mm diameter software aperture, producing a final in-band residual of $0.57 \mathrm{~nm}$ RMS. This shape was used to measure the reference slopes. The reference slopes should account for the $27 \mathrm{~nm}$ due to CCD misalignment, as well as the $13 \mathrm{~nm}$ which are left over from other sources, for a total of $30 \mathrm{~nm}$ of NCP error.

The WFS-FTR system was run in closed-loop until the system converged. The PSDI was then used to measure the residual phase error in the same $9.2 \mathrm{~mm}$ aperture. This residual error was $1.00 \mathrm{~nm}$ RMS in band. This residual error, low-pass filtered, is shown in Fig. 5, left panel. The black lump in the center is due to a misbehaving actuator. The two dark lumps at the very top of the aperture are due to two pinned actuators directly above. These mispositionings contribute substantially to the total RMS error. The spatial PSD of the full signal is shown in the right side of the figure. The in-band residual error has small low-order term but is dominated by a high-spatial-frequency term near the edge of the controllable region. (The four bright lumps are the ripple on the actuators at spatial frequency $1 / d$.) This residual error is dominated by a highspatial-frequency term which remains constant across different references and trials, which indicates a static $\mathbf{e}_{\text {ref }}$ in reference measurement. We will compensate for this error in reference measurement using the PSDI as a calibration system. 

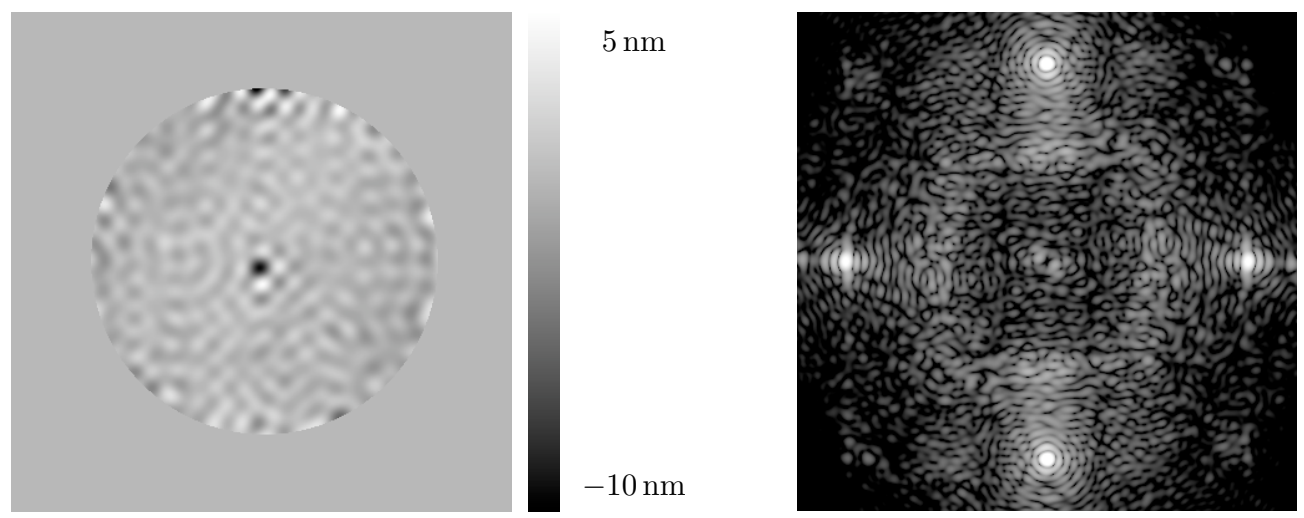

Figure 5. [left] Lowpass-filtered residual phase error on the MEMS after flattening with WFS-FTR with best alignment and references. In this $9.2 \mathrm{~mm}$ aperture (27 subapertures across), the in-band RMS error is $1.00 \mathrm{~nm}$. [right] Spatial power spectrum of this residual error, log-color scale. This residual error is dominated by error at the edge of the controllable band and has a small low-order term.
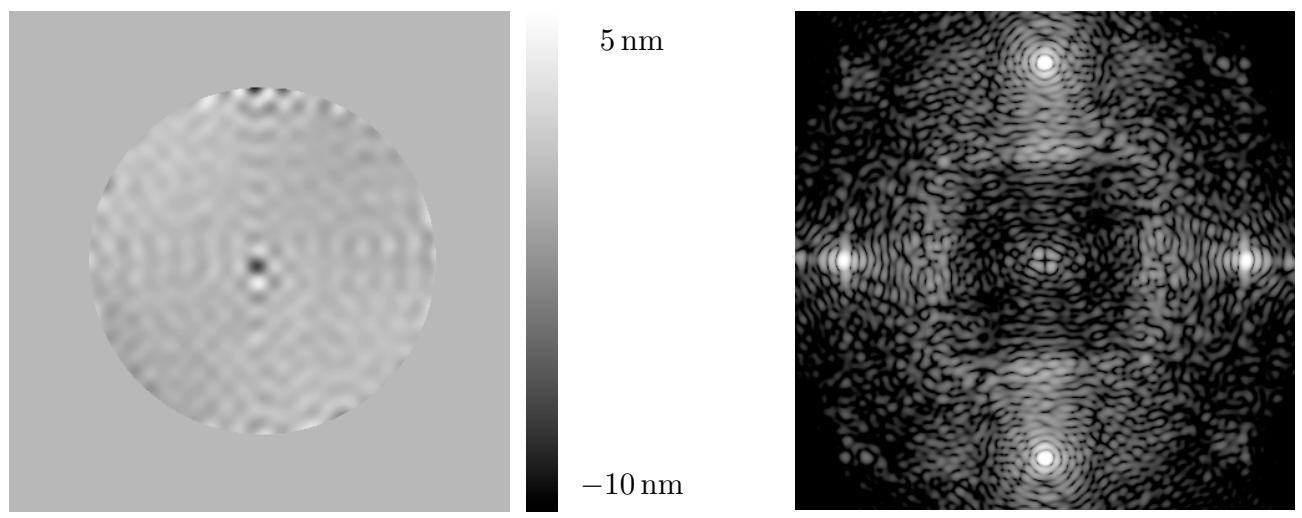

Figure 6. [left] Lowpass-filtered residual phase error on the MEMS after a single-step adjustment of the references, using the calibration system algorithm with the PSDI measurement of Fig. 5. This is $0.70 \mathrm{~nm}$ RMS in-band. [right] Spatial power spectrum of this residual error, log-color scale. Most of the correction occurred at high spatial frequencies.

\section{USE OF THE CALIBRATION SYSTEM}

The calibration system measures the static (or quasi-static, depending on the time scale of the measurement) phase aberration in the science leg. This static error can be caused because the system calibration was measured incorrectly, or because the system has physically changed (e.g. due to flexure on the optical board). If this static aberration is within the controllable band of the WFS, the information from the calibration system can be incorporated into the reference slopes in a manner that fixes this static error.

As shown above, some specific phase aberrations in the science leg have slopes which are easy to determine analytically. In the general case, a Fourier filter can be used to calculate the slopes, ${ }^{5}$ based on the frequency response of an ideal Shack-Hartmann WFS. Our algorithm for determining the new reference slopes begins with a PSDI measurement of the residual phase error. This is down-sampled and aligned with the actuator grid. Given this in-band estimate of the phase at the actuators, the Shack-Hartmann filter is applied. The resulting slopes (in nm of phase slope per subaperture) are added directly to the existing reference slopes.

This was done for the residual error shown in Fig. 5, with no masking of any of the misbehaving actuators. The WFS-FTR closed loop was run with these modified reference slopes and after convergence, a PSDI measurement was taken. This is shown in the left side of Fig. 6 . The new residual error is $0.70 \mathrm{~nm}$ RMS in-band, which means that the single slope adjustment step from calibration system information removed about $0.70 \mathrm{~nm}$ RMS 
in-band of error which was due to incorrectly measured reference slopes. Most of this error was in the highspatial-frequencies near the edge of the controllable region, though some was a the lowest spatial frequencies.

As an additional test, we iterated on this calibration step one more time. The second time, the residual in Fig. 6 was used as input to the reference adjustment algorithm. Masking out the misbehaving center actuator before applying the algorithm resulted in references which produced a closed-loop residual error to just $0.64 \mathrm{~nm}$ RMS in band.

\section{CONCLUSIONS}

We have demonstrated some fundamental techniques to our advanced GPI wavefront control strategy. In particular, we have shown that with proper alignment and referencing using only WFS information, the SFWFS-FTR combination can flatten the $32 \times 32$ MEMS device to $1 \mathrm{~nm}$ RMS in-band error on its surface. In addition, a high-accuracy measurement of the residual static error in the science leg (provided to us by the PSDI) can be used to modify the references and significantly improves flattening performance to $0.7 \mathrm{~nm}$ RMS in-band.

Further work remains to be done. Now that we have demonstrated $1 \mathrm{~nm}$ RMS in-band flattening of the MEMS, we want to extend this to correction of atmosphere-like phase aberrations. Tests on manufactured phase plates will begin in May 2006. Much work also remains on characterizing the calibration system model of converting residual phase errors in the science leg into reference slope modifications. In particular, we need to explore temporal filtering to ensure stability in reference modification and realistic signal-to-noise and accuracy considerations for the true calibration system in GPI.

We also want to track down the cause of the incorrect initial reference measurements, which appear to be dominated by high-spatial-frequency error. A possible culprits is the physical spatial filter, which is not manufactured to the exact specification for size and corner shape. Another possibility is that the Vitrum lenslet array is not exactly in a conjugate plane to the MEMS mirror; this lack of conjugation will cause the phase to be slightly different in the lenslet plane, which may be the source of the error.

\section{ACKNOWLEDGMENTS}

Thanks to Julia W. Evans for her insights on the system performance when the PSDI is used for flattening, and her flexibility is sharing experimental time on the testbed. This work was performed under the auspices of the U.S. Department of Energy by the University of California, Lawrence Livermore National Laboratory under contract No. W-7405-Eng-48. The document number is UCRL-CONF-220694. This work has been supported in part by the National Science Foundation Science and Technology Center for Adaptive Optics, managed by the University of California at Santa Cruz under cooperative agreement No. AST-9876783 and in part by the Gordon and Betty Moore Foundation through its grant to the UCO/Lick Laboratory for Adaptive Optics.

\section{REFERENCES}

1. B. A. Macintosh and et al, "The Gemini Planet Imager," in Advances in Adaptive Optics II, B. L. Ellerbroek and D. B. Calia, eds., Proc SPIE 6272, p. this volume, 2006.

2. L. A. Poyneer and B. Macintosh, "Spatially filtered wave-front sensor for high-order adaptive optics," J. Opt. Soc. Am. A 21, pp. 810-819, 2004.

3. L. A. Poyneer, B. Bauman, B. A. Macintosh, D. Dillon, and S. Severson, "Experimental demonstration of phase correction with a $32 \times 32$ microelectricalmechanical systems mirror and a spatially filtered wavefront sensor," Opt. Lett. 31, pp. 293-295, 2006.

4. L. A. Poyneer, D. T. Gavel, and J. M. Brase, "Fast wave-front reconstruction in large adaptive optics systems with use of the Fourier transform," J. Opt. Soc. Am. A 19, pp. 2100-2111, 2002.

5. L. A. Poyneer and J.-P. Véran, "Optimal modal fourier transform wave-front control," J. Opt. Soc. Am. A 22, pp. 1515-1526, 2005.

6. J. K. Wallace, J. J. Green, M. Shao, M. Troy, J. P. Lloyd, and B. Macintosh, "Science camera calibration for extreme adaptive optics," in Advancements in Adaptive Optics, D. B. Calia, B. L. Ellerbroek, and R. Ragazzoni, eds., Proc SPIE 5490, pp. 370-378, 2004. 
7. M. Nicolle, T. Fusco, G. Rousset, and V. Michaut, "Improvement of Shack-Hartmann wave-front sensor measurement for extreme adaptive optics," Opt. Lett. 29, pp. 2743-2745, 2004.

8. J.-P. Véran and G. Herriot, "Centroid gain compensation in Shack-Hartmann adaptive optics systems with natural or laser guide star," J. Opt. Soc. Am. A 17(1430-1439), 2002.

9. B. A. Macintosh, B. Bauman, J. W. Evans, J. R. Graham, C. Lockwood, L. Poyneer, D. Dillon, D. T. Gavel, J. J. Green, J. P. Lloyd, R. B. Makidon, S. Olivier, D. Palmer, M. D. Perrin, S. Severson, A. I. Sheinis, A. Sivaramakrishnan, G. Sommargren, R. Soummer, M. Troy, J. K. Wallace, and E. Wishnow, "eXtreme adaptive optics planet imager: overview and status," in Advancements in Adaptive Optics, D. B. Calia, B. L. Ellerbroek, and R. Ragazzoni, eds., Proc. SPIE 5490, pp. 359-369, 2004.

10. A. Sivaramakrishnan, J. P. Lloyd, P. Hodge, and B. A. Macintosh, "Speckle decorrelation and dynamic range in speckle noise-limited imaging," Ap. J. 581, pp. L59-62, 2002.

11. M. D. Perrin, A. Sivaramakrishnan, R. B. Makidon, B. R. Oppenheimer, and J. R. Graham, "The structure of high strehl ratio point-spread functions," Ap. J. 596, pp. 702-712, 2003.

12. B. L. Ellerbroek, "Efficient computation of minimum-variance wave-front reconstructors with sparse matrix techniques," J. Opt. Soc. Am. A 19, pp. 1803-1816, 2002.

13. J.-P. Véran and G. Herriot, "Woofer-tweeter tip-tilt control for NFIRAOS on TMT," in Advances in Adaptive Optics II, B. L. Ellerbroek and D. B. Calia, eds., Proc SPIE 6272, p. this volume, 2006.

14. L. K. Saddlemyer, G. Herriot, and J.-P. Veran, "Design and current status of the reconstructor for Altair: the Gemini North adaptive optics system," in Adaptive Optical Systems Technology, P. L. Wizinowich, ed., Proc SPIE 4007, pp. 649-658, 2000.

15. J. W. Evans, G. Sommargren, L. Poyneer, B. A. Macintosh, S. Severson, D. Dillon, A. I. Sheinis, D. Palmer, N. J. Kasdin, and S. Olivier, "Extreme adaptive optics testbed: results and future work," in Advancements in Adaptive Optics, D. B. Calia, B. L. Ellerbroek, and R. Ragazzoni, eds., Proc. SPIE 5490, pp. 954-959, 2004.

16. G. E. Sommargren, D. W. Phillion, M. A. Johnson, N. Q. Nguyen, A. Barty, F. J. Snell, D. R. Dillon, and L. S. Bradsher, "100-picometer interferometry for EUVL," in Emerging Lithographic Technologies V, R. L. Engelstad, ed., Proc. SPIE 4688, pp. 316-328, 2002.

17. L. A. Poyneer, D. W. Palmer, K. N. LaFortune, and B. Bauman, "Experimental results for correlationbased wavefront sensing," in Advanced Wavefront Control: Methods, Devices, and Applications III, M. T. Gruneisen, J. D. Gonglewski, and M. K. Giles, eds., Proc. SPIE 5894, p. 58940N, 2005.

18. C. Marois, R. Doyon, D. Nadeau, R. Racine, M. Riopel, P. Vallée, and D. Lafrenière, "TRIDENT: An Infrared Differential Imaging Camera Optimized for the Detection of Methanated Substellar Companions," Publ. Astr. Soc. Pac. 117, pp. 745-756, 2005.

19. D. S. Acton, P. L. Wizinowich, J. Paul J. Stomski, J. C. Shelton, O. Lai, and J. M. Brase, "Laboratory calibration of the W.M. Keck Observatory adaptive optics facility," in Adaptive Optical System Technologies, D. Bonaccini and R. K. Tyson, eds., Proc SPIE 3353, pp. 125-131, 1998.

20. J. W. Evans, B. Macintosh, L. A. Poyneer, K. Morzinski, S. Severson, D. Dillon, D. Gavel, and L. Reza, "Demonstrating sub-nm closed loop MEMS flattening," Opt. Exp., submitted 2006.

21. K. N. LaFortune, R. L. Hurd, E. M. Johansson, C. B. Dane, S. N. Fochs, and J. M. Brase, "Intracavity adaptive correction of a 10-kW solid state heat-capacity laser," in Laser Resonators and Beam Control VII, A. V. Kudryashov, ed., Proc. SPIE 5333, pp. 53-61, 2004.

22. M. D. Oliker, "Alignment techniques for DM, lenslet, and WFS camera at the SOR," in Adaptive Optics and Applications, R. K. Tyson and R. Q. Fugate, eds., Proc SPIE 3126 3126, pp. 595-604, 1997.

23. J. W. Evans, K. Morzinski, L. Reza, S. Severson, L. Poyneer, B. Macintosh, D. Dillon, G. Sommargren, D. Palmer, D. Gavel, and S. Olivier, "Extreme adaptive optics testbed: High contrast measurements with a mems deformable mirror," in Techniques and Instrumentation for Detection of Exoplanets II, D. R. Coulter, ed., Proc. SPIE 5905, p. 59050Y, 2005. 